\title{
Health-related problems and quality of life in patients with syndromic and complex craniosynostosis
}

\author{
Tim de Jong • Marianne Maliepaard • \\ Natalja Bannink • Hein Raat • Irene M. J. Mathijssen
}

Received: 12 December 2011 / Accepted: 2 January 2012 / Published online: 11 January 2012

(C) The Author(s) 2012. This article is published with open access at Springerlink.com

\begin{abstract}
Purpose We conducted this study to gauge the health-related problems, quality of life and the performance of the Health Utility Index Mark 3 (HUI-3) in patients with syndromic and complex craniosynostosis. Patients with syndromic and complex craniosynostosis have various physical and mental problems. More insight on these problems, per syndrome, could provide guidance to improve patient treatment and follow-up. Methods A cross-sectional, comparative study on 131 patients and their parents was performed. Health-related quality of life was measured with the HUI-3 and the Visual Analogue Scale (VAS). All data were compared to a normative Dutch population. Vision, hearing and intelligence were objectively measured.

Results The HUI-3 and the VAS were significant lower compared to the normative Dutch population. All syndromes have a high prevalence of vision and speech problems. Cognitive problems were mainly reported in patients with Apert, Crouzon and Muenke syndrome. Ambulation and dexterity problems were seen in Apert, Crouzon, Saethre-Chotzen and complex craniosynostosis. Only patients with Apert syndrome scored significantly worse on pain. The HUI-3 had a medium to strong correlation with the objectively measured outcomes. Conclusions The overall quality of life is lower in patients with syndromic and complex craniosynostosis. To improve
\end{abstract}

T. de Jong $(\varangle) \cdot$ M. Maliepaard $\cdot$ N. Bannink $\cdot$ I. M. J. Mathijssen

Department of Plastic and Reconstructive Surgery and Hand

Surgery, Dutch Craniofacial Center, Erasmus MC,

Sophia Children's Hospital,

Room Ee 15.91, Dr. Molewaterplein 50,

3015 GE, Rotterdam, The Netherlands

e-mail: t.dejong@erasmusmc.nl

H. Raat

Department of Public Health, Erasmus MC,

Rotterdam, The Netherlands quality of life, more attention is needed for problems with vision and speech.

Keywords Craniosynostosis · Quality of life · Health Utility Index · Visual Analogue Scale

\section{Introduction}

Craniosynostosis involves the premature closure of the cranial sutures. In about $40 \%$ of the patients, it is part of a syndrome such as Apert, Crouzon, Muenke and Saethre-Chotzen. Patients with syndromic and complex craniosynostosis have a lower health related quality of life (HRQoL), while patients with isolated craniosynostosis score within the normal range for quality of life and behavioral problems [3]. Reasons for the lower HRQoL are problems concerning physical functioning, bodily pain and mental health [1]. Commonly reported healthrelated problems in syndromic craniosynostosis are hearing and visual disorders, sleep apnea and hand and foot anomalies [11]. The prevalence and severity of these problems vary per syndrome, and it is unknown to what extent they influence the HRQoL and parents perceived quality of life. The aim of this study was to evaluate health related problems, quality of life and the performance of the Health Utility Index Mark 3 (HUI-3) in patients with syndromic craniosynostosis.

\section{Material and methods}

A cross-sectional comparative study was performed in patients 4-18 years of age, with a diagnosis of syndromic or complex craniosynostosis. All patients were treated at the craniofacial unit of a tertiary pediatric hospital. Patients were included in the study if they had craniosynostosis 
associated with Apert, Crouzon, Pfeiffer, Saethre-Chotzen, Muenke syndrome or complex craniosynostosis. Syndrome diagnosis was based on genetic testing. Complex craniosynostosis was defined as the premature closure of two or more sutures in the absence of a genetic mutation. Because Crouzon and Pfeiffer syndrome cannot be distinguished from each other genetically, they were considered a homogeneous group in this study.

The health-related quality of life was assessed using the HUI-3 questionnaire [13, 14]. The HUI is developed to measure health-related quality of life and is applicable in clinical studies. The HUI is suitable for patients of 5 years and older, and for children under the age of 8 years a proxy assessment is recommended. Because we approached a large group of patients younger than 8 years and there is a high prevalence of cognitive impairment in patients with syndromic craniosynostosis, we requested the parents to complete the questionnaire. Patients were compared to normative data from a general Dutch population survey. ${ }^{15}$ It were also the parents who completed the questionnaire in the Dutch population survey. Based on the questionnaire, subjects were classified according to the HUI-3 classification system. The HUI is a utility (preference) based scoring system for measuring comprehensive health status and health-related quality of life, consisting of eight attributes. Each attribute was scored from 1 (no limitations) to 4, 5 or 6 (severe limitations). Single attribute utility scores range from 1.00 to 0.00 , where perfect health is 1.00 and dead is 0.00 . Multi-attribute utility scores, indicating overall health, are calculated based on single attribute scores $[8,18]$. The multi-attribute HUI score can be negative, which indicates a state described as worse than dead. Next to the HUI, the Visual Analogue Scale (VAS) was used to rate parent-perceived overall health of the child, with a score ranging from 0 (worst health) to 100 (best health). The questionnaire was once sent by mail once.

From the eight attributes of the HUI-3, vision, hearing and intelligence can be measured objectively. Therefore, we collected data of vision, hearing and intelligence to compare to the corresponding HUI-3 attributes. Data of vision was retrospectively collected. Vision is routinely checked with a Snellen chart by our pediatric ophthalmologist. Data of hearing was cross-sectionally gathered as part of another study [5]. Hearing was tested by a pediatric audiologist with pure tone audiometry. Hearing loss was expressed as the average hearing loss at 500, 1,000 and 2,000 $\mathrm{Hz}$ of the best ear. Intelligence was tested by a pediatric psychologist with the Wechsler Intelligence Scale for Children (WISC)-III, as part of a prospective study.

\section{Statistical analysis}

Mann-Whitney $U$-test was used to compare means within the syndrome groups and between the syndrome groups and the normative population. Pearson's chi-square test was used to test correlation. A two-sided $p \leq 0.05$ was considered significant. All analyses were performed using SPSS 16.0 (SPSS Inc., Chicago, IL, USA).

\section{Results}

A total of 173 patients were approached, of whom 131 responded (76\%). Of the 131 patients, 20 had Apert, 39 Crouzon/Pfeiffer, 25 Muenke, 18 Saethre-Chotzen and 29 complex craniosynostosis. The mean age of the patients at the time of this review was 9.6 years, and $45 \%$ were female. The average age of the respondents was 41.6 years, and $73 \%$ were female (see Table 1). There was no significant difference between how mothers and fathers scored the HUI- 3 and VAS, in the normative Dutch population and craniosynostosis groups. Age and gender did not influence quality of life, except for Apert syndrome, were the males had a significant lower HUI-3.

The percentage of patients who are not affected and the mean single attribute HUI- 3 scores are shown in Table 2. Patients were considered to be not affected if they had the best possible score for that specific attribute. Vision, speech and cognition were the most affected attributes in patients with syndromic craniosynostosis. Speech was significant associated with hearing $(p<0.001)$ and cognition $(p<0.001)$.

The mean VAS and multi-attribute HUI-3 score are shown in Table 3. There was no significant difference between the multi-attribute score and the VAS except for the Apert syndrome, in whom the HUI-3 was significant lower. This difference indicates that parents subjectively experience a higher

Table 1 Characteristics of patients and the normative population

\begin{tabular}{|c|c|c|c|c|c|c|}
\hline & $\begin{array}{l}\text { Apert } \\
(n=20)\end{array}$ & $\begin{array}{l}\text { Crouzon } \\
(n=39)\end{array}$ & $\begin{array}{l}\text { Muenke } \\
(n=25)\end{array}$ & $\begin{array}{l}\text { Saethre- } \\
\text { Chotzen } \\
(n=18)\end{array}$ & $\begin{array}{l}\text { Complex } \\
(n=29)\end{array}$ & $\begin{array}{l}\text { Norm group } \\
(n=1435)\end{array}$ \\
\hline Sex patient $\mathrm{M} / \mathrm{F}$ & $8 / 12$ & $18 / 21$ & $11 / 14$ & $5 / 13$ & $17 / 12$ & $708 / 727$ \\
\hline Age patients in years, mean (SD) & $10.6(4.9)$ & $9.5(3.9)$ & $9.3(4.1)$ & $10.4(3.9)$ & $8.7(4.0)$ & $8.1(2.4)$ \\
\hline Sex parent $\mathrm{M} / \mathrm{F}$ & $6 / 14$ & $9 / 30$ & $6 / 19$ & $4 / 14$ & $7 / 22$ & $206 / 1229$ \\
\hline Age parent in years, mean (SD) & $41.6(8.0)$ & $41.8(5.3)$ & $40.5(6.3)$ & $43.3(7.9)$ & $41.3(6.0)$ & $37.7(5.2)$ \\
\hline
\end{tabular}


Table 2 Mean single-attribute HUI-3 of patients and the normative population

\begin{tabular}{|c|c|c|c|c|c|c|c|c|c|c|c|c|}
\hline \multirow[b]{2}{*}{ HUI-3 } & \multicolumn{2}{|c|}{ Apert $(n=20)$} & \multicolumn{2}{|c|}{ Crouzon $(n=39)$} & \multicolumn{2}{|c|}{ Muenke $(n=25)$} & \multicolumn{2}{|c|}{$\begin{array}{l}\text { Saethre-Chotzen } \\
(n=18)\end{array}$} & \multicolumn{2}{|c|}{ Complex $(n=29)$} & \multicolumn{2}{|c|}{$\begin{array}{l}\text { Norm group } \\
(n=1435)\end{array}$} \\
\hline & $\begin{array}{l}\% \text { not } \\
\text { affected }\end{array}$ & Mean & $\begin{array}{l}\% \text { not } \\
\text { affected }\end{array}$ & Mean & $\begin{array}{l}\% \text { not } \\
\text { affected }\end{array}$ & Mean & $\begin{array}{l}\% \text { not } \\
\text { affected }\end{array}$ & Mean & $\begin{array}{l}\% \text { not } \\
\text { affected }\end{array}$ & Mean & $\begin{array}{l}\% \text { not } \\
\text { affected }\end{array}$ & Mean \\
\hline Vision & 50 & $0.90^{ \pm}$ & 50 & $0.90^{ \pm}$ & 60 & $0.97^{ \pm}$ & 31 & $0.96^{ \pm}$ & 72 & $0.99^{ \pm}$ & 93 & 0.99 \\
\hline Hearing & 85 & $0.93^{ \pm}$ & 79 & $0.93^{ \pm}$ & 76 & $0.93^{ \pm}$ & 89 & $0.96^{\#}$ & 93 & $0.98^{*}$ & 98 & 1.00 \\
\hline Speech & 20 & $0.68^{ \pm}$ & 56 & $0.84^{ \pm}$ & 56 & $0.84^{ \pm}$ & 71 & $0.95^{*}$ & 66 & $0.87^{ \pm}$ & 88 & 0.97 \\
\hline Ambulation & 85 & $0.97^{ \pm}$ & 90 & $0.96^{ \pm}$ & 100 & 1.00 & 100 & 1.00 & 93 & $0.96^{ \pm}$ & 99 & 1.00 \\
\hline Dexterity & 0 & $0.58^{ \pm}$ & 90 & $0.95^{ \pm}$ & 100 & 1.00 & 94 & $0.98^{\#}$ & 93 & $0.96^{ \pm}$ & 99 & 1.00 \\
\hline Emotion & 80 & 0.97 & 71 & 0.97 & 71 & 0.97 & 76 & 0.98 & 83 & 0.98 & 81 & 0.98 \\
\hline Cognition & 25 & $0.77^{ \pm}$ & 61 & $0.93^{ \pm}$ & 72 & $0.89^{*}$ & 78 & 0.97 & 72 & 0.94 & 84 & 0.97 \\
\hline Pain & 55 & $0.93^{\#}$ & 73 & 0.97 & 76 & 0.96 & 76 & 0.98 & 79 & 0.97 & 81 & 0.98 \\
\hline
\end{tabular}

${ }^{*} p<0.05,{ }^{*} p<0.01,{ }^{ \pm} p<0.001$ compared to norm group

quality of life for their child than their objective scoring indicates. The correlation between the multi-attribute HUI-3 and the VAS was $0.476(p<0.001)$ in patients with craniosynostosis.

Of the 131 included patients, $68(52 \%)$ had data on vision, $63(48 \%)$ on hearing and $60(46 \%)$ were tested with the WISC-III. The correlation between the objective measurements and the single-attributes was $0.558(p<0.001)$ for vision, $-0.345(p=0.006)$ for hearing and $0.418(p=0.001)$ for intelligence.

\section{Discussion}

The multi-attribute HUI-3 and the VAS are significantly lower in patients with craniosynostosis compared to the normative Dutch population. The main reasons for this are problems with vision and speech, and cognition in Apert and Crouzon syndrome. Ambulation, emotional problems and pain have no or limited influence on the HRQoL in syndromic craniosynostosis. In general, age and gender did not influence the quality

Table 3 Mean multi-attribute score of the HUI-3 and VAS

\begin{tabular}{lll}
\hline & HUI-3 & VAS \\
\hline Apert $(n=20)$, mean (SD) & $0.44^{*}(0.30)$ & $0.77(0.20)$ \\
Crouzon $(n=39)$, mean (SD) & $0.76(0.23)$ & $0.79(0.18)$ \\
Muenke $(n=25)$, mean (SD) & $0.81(0.22)$ & $0.81(0.16)$ \\
Saethre-Chotzen $(n=18)$, mean (SD) & $0.87(0.14)$ & $0.88(0.11)$ \\
Complex $(n=29)$, mean (SD) & $0.83(0.24)$ & $0.87(0.10)$ \\
Total craniosynostosis group & $0.75(0.27)^{ \pm}$ & $0.82(0.16)^{ \pm}$ \\
$\quad(n=132)$, mean (SD) & $0.91(0.12)$ & $0.93(0.09)$ \\
\hline Norm group $(n=1435)$, mean (SD) & 0 &
\end{tabular}

* Significantly lower compared to VAS, $p<0.05$

${ }^{ \pm}$Significantly lower compared to norm group, $p<0.001$ of life. The overall correlation between the objective measurements and the HUI-3 attributes is medium to strong.

Most problems were found in the attributes vision, speech and cognition, despite routine screening for these impairments in our patients. This screening and treatment is thus essential but cannot prevent or overcome all restrictions. Problems with vision affect about half the patients and can be due to refractive error, strabismus, astigmatism and persistent elevated intracranial pressure [2, 4, 10, 12]. Speech problems have previous been reported in Apert, Muenke and Saethre-Chotzen syndrome [6, 7, 17]. Causes for speech problems are hearing deficits, oral anomalies, learning disabilities and impaired social interaction [17]. The impaired cognition is probably not the main reason for speech problems but will contribute to a worse language development [17]. The level of intelligence varies strongly per syndrome but also within every syndrome, especially patients with Apert syndrome can have a low intelligence [9, 15, 19, 20].

The single-attribute HUI-3 had a medium to strong correlation with the objectively measured vision, hearing and intelligence. However, in individual cases, there were large differences between how parents scored the vision, hearing or intelligence and the objective measurements. It is known that parents have difficulty judging their child's hearing [16]. Therefore, we conclude that the HUI-3 is less suitable in individual patients for follow-up, but can be used on a group level for patients with syndromic craniosynostosis.

A limitation of this study is that although this is one of the largest reported groups of children with syndromic craniosynostosis, the syndrome-specific groups still have a small sample size, making comparisons within groups not more than explanatory. Another limitation is the crosssectional design of this study.

In conclusion, the overall quality of life is lower in patients with syndromic and complex craniosynostosis. To improve quality of life more attention is needed for problems 
with vision and speech. As there can be a big discrepancy between objective measures and how parents score the HUI-3, this questionnaire is more suitable for groups than for individual follow-up.

The Erasmus Medical Center medical ethical review board approved the study under reference number: MEC-2005-273.

\section{Acknowledgment This study was funded by the Carolien Bijl} foundation.

Open Access This article is distributed under the terms of the Creative Commons Attribution Noncommercial License which permits any noncommercial use, distribution, and reproduction in any medium, provided the original author(s) and source are credited.

\section{References}

1. Bannink N, Maliepaard M, Raat H, Joosten KF, Mathijssen IM (2010) Health-related quality of life in children and adolescents with syndromic craniosynostosis. J Plast Reconstr Aesthet Surg. S1748-S6815

2. Bartels MC, Vaandrager JM, de Jong TH, Simonsz HJ (2004) Visual loss in syndromic craniosynostosis with papilledema but without other symptoms of intracranial hypertension. J Craniofac Surg 15(6):1019-1022

3. Boltshauser E, Ludwig S, Dietrich F, Landolt MA (2003) Sagittal craniosynostosis: cognitive development, behaviour, and quality of life in unoperated children. Neuropediatrics 34(6):293-300

4. de Jong T, Bannink N, Bredero-Boelhouwer HH, van Veelen ML, Bartels MC, Hoeve LJ, Hoogeboom AJ, Wolvius EB, Lequin MH, van der Meulen JJ, van Adrichem LN, Vaandrager JM, Ongkosuwito EM, Joosten KF, Mathijssen IM (2009) Long-term functional outcome in 167 patients with syndromic craniosynostosis; defining a syndrome-specific risk profile. J Plast Reconstr Aesthet Surg. S1748-S6815

5. de Jong T, Toll MS, de Gier HH, Mathijssen IM (2011) Audiological profile of children and young adults with syndromic and complex craniosynostosis. Arch Otolaryngol Head Neck Surg 137(8):775-778

6. Doherty ES, Lacbawan F, Hadley DW, Brewer C, Zalewski C, Kim HJ, Solomon B, Rosenbaum K, Domingo DL, Hart TC, Brooks BP, Immken L, Lowry RB, Kimonis V, Shanske AL, Jehee FS, Bueno MR, Knightly C, McDonald-McGinn D, Zackai EH, Muenke M (2007) Muenke syndrome (FGFR3-related craniosynostosis): expansion of the phenotype and review of the literature. Am J Med Genet A 143A(24):3204-3215
7. Elfenbein JL, Waziri M, Morris HL (1981) Verbal communication skills of six children with craniofacial anomalies. Cleft Palate J 18 (1):59-64

8. Feeny D, Furlong W, Torrance GW, Goldsmith $\mathrm{CH}$, Zhu Z, DePauw S, Denton M, Boyle M (2002) Multiattribute and single-attribute utility functions for the Health Utilities Index Mark 3 system. Med Care 40(2):113-128

9. Flapper WJ, Anderson PJ, Roberts RM, David DJ (2009) Intellectual outcomes following protocol management in Crouzon, Pfeiffer, and Muenke syndromes. J Craniofac Surg 20(4):1252-1255

10. Jadico SK, Huebner A, McDonald-McGinn DM, Zackai EH, Young TL (2006) Ocular phenotype correlations in patients with TWIST versus FGFR3 genetic mutations. J AAPOS 10(5):435-444

11. Johnson D, Wilkie AO (2011) Craniosynostosis. Eur J Hum Genet 19(4):369-376

12. Khong JJ, Anderson P, Gray TL, Hammerton M, Selva D, David D (2006) Ophthalmic findings in apert syndrome prior to craniofacial surgery. Am J Ophthalmol 142(2):328-330

13. Raat H, Bonsel GJ, Essink-Bot ML, Landgraf JM, Gemke RJ (2002) Reliability and validity of comprehensive health status measures in children: The Child Health Questionnaire in relation to the Health Utilities Index. Journal of clinical epidemiology 55 (1):67-76

14. Raat H, Bonsel GJ, Hoogeveen WC, Essink-Bot ML (2004) Feasibility and reliability of a mailed questionnaire to obtain visual analogue scale valuations for health states defined by the Health Utilities Index Mark 3. Medical Care 42(1):13-18

15. Renier D, Arnaud E, Cinalli G, Sebag G, Zerah M, Marchac D (1996) Prognosis for mental function in Apert's syndrome. Journal Neurosurg 85(1):66-72

16. Rosenfeld RM, Goldsmith AJ, Madell JR (1998) How accurate is parent rating of hearing for children with otitis media? Arch Otolaryngol Head Neck Surg 124(9):989-992

17. Shipster C, Hearst D, Dockrell JE, Kilby E, Hayward R (2002) Speech and language skills and cognitive functioning in children with Apert syndrome: a pilot study. Int J Lang Commun Disord 37 (3):325-343

18. Torrance GW, Feeny DH, Furlong WJ, Barr RD, Zhang Y, Wang Q (1996) Multiattribute utility function for a comprehensive health status classification system. Health Utilities Index Mark 2. Med Care 34(7):702-722

19. Yacubian-Fernandes A, Ducati LG, Silva MV, Abramides DV, Perosa GB, Palhares A, Gabarra RC, Giglio A, Portela L, Marinello JL, Plese JP, Zanini SA (2007) Crouzon syndrome: factors related to the neuropsychological development and to the quality of life. Arq Neuropsiquiatr 65(2B):467-471

20. Yacubian-Fernandes A, Palhares A, Giglio A, Gabarra RC, Zanini S, Portela L, Silva MV, Perosa GB, Abramides D, Plese JP (2005) Apert syndrome: factors involved in the cognitive development. Arq Neuropsiquiatr 63(4):963-968 